\title{
Lupeol reduces M1 macrophage polarization to attenuate immunologic dissonance and fatty acid deposition in rats with diet-induced metabolic syndrome
}

\author{
Jin Li ${ }^{1 \#}$, Yuechen Huang ${ }^{2 \#}$, Yue Han ${ }^{3}$, Jiafu Wang ${ }^{4}$, Chun Zhang', Jiuyang Jiang ${ }^{1}$ \\ ${ }^{1}$ Department of Traditional Chinese Medicine, The First Affiliated Hospital of Harbin Medical University, Harbin, China; ${ }^{2}$ Boli County Hospital of \\ Traditional Chinese Medicine, Qitaihe, China; ${ }^{3}$ Department of Ultrasound Medicine, The First Affiliated Hospital of Harbin Medical University, \\ Harbin, China; ${ }^{4}$ Department of Nuclear Medicine, The First Affiliated Hospital of Harbin Medical University, Harbin, China \\ Contributions: (I) Conception and design: J Li, Y Huang; (II) Administrative support: J Li, Y Han, J Jiang; (III) Provision of study materials or \\ patients: J Li, C Zhang; (IV) Collection and assembly of data: Y Huang, C Zhang, J Jiang; (V) Data analysis and interpretation: Y Huang, Y Han; (VI) \\ Manuscript writing: All authors; (VII) Final approval of manuscript: All authors. \\ \#These authors contributed equally to this work. \\ Correspondence to: Jiuyang Jiang. Department of Traditional Chinese Medicine, The First Affiliated Hospital of Harbin Medical University, Harbin, \\ China. Email: a13603685799@163.com.
}

Background: This study aimed to investigate whether lupeol could inhibit the inflammatory mediators associated with the regulation of macrophage phenotypes and functions in rats with diet-induced metabolic syndrome (MS).

Methods: Forty specific-pathogen-free Sprague Dawley rats were fed a high-fat diet (HFD) for 10 weeks to establish an MS model. Lupeol was prepared and administered to the rats intraperitoneally at 20, 50, or $100 \mathrm{mg} / \mathrm{kg}$ (the lupeol $20 \mathrm{mg} / \mathrm{kg}$, lupeol $50 \mathrm{mg} / \mathrm{kg}$, and lupeol $100 \mathrm{mg} / \mathrm{kg}$ groups respectively). After 28 days of continuous intraperitoneal administration, rats were anesthesia and euthanasia. The obesity index, blood glucose and lipid metabolism indexes of rats in each group were measured. The levels of insulin and inflammatory factors in each group were detected by enzyme-linked immunosorbent assay (ELISA) kits. The pathological changes of liver tissue in rats were observed by hematoxylin and eosin (HE) and oil red $\mathrm{O}$ staining. The polarization levels of M1 and M2 macrophages in peripheral blood mononuclear cells (PBMCs) were analyzed by flow cytometry. The transcription levels of M1 and M2 macrophages markers were detected by qRT-PCR. The expressions of inducible nitric oxide synthase (iNOS) and arginase-1 (Arg-1) proteins in heart tissues of rats in each group were analyzed by Western blotting.

Results: Lupeol significantly recovered fasting blood glucose and serum insulin levels, and reduced the production of proinflammatory cytokines, including interleukin (IL)-6, tumor necrosis factor alpha (TNF- $\alpha$ ), and monocyte chemoattractant protein-1 (MCP-1), in the liver. It also elevated the expression of antiinflammatory cytokines, including IL-4 and IL-10, in the MS model. Further, after treatment with lupeol, the levels of total cholesterol, triacylglycerol and low-density lipoprotein (LDL) were decreased, and highdensity lipoprotein (HDL) were increased. Importantly, in the MS model group, lupeol remarkably inhibited M1 macrophages polarization $\left(\mathrm{F} 4 / 80^{+} \mathrm{iNOS}^{+}\right.$) while elevating $\mathrm{M} 2$ macrophages polarization $\left(\mathrm{F} 4 / 80^{+} \mathrm{CD} 206^{+}\right)$ remarkably. At the same time, the levels of M1 markers, including inducible nitric oxide synthase, IL- $1 \beta$, IL6 , and TNF- $\alpha$, were markedly inhibited, while those of M2 markers, such as arginase-1, IL-10, CD206, and TGF- $\beta$, were markedly elevated in the MS model rats.

Conclusions: Lupeol might promote M2 polarization of macrophages to relieve damage caused by MS.

Keywords: Lupeol; metabolic syndrome (MS); M1 macrophage; M2 macrophage

Submitted Aug 06, 2021. Accepted for publication Oct 13, 2021.

doi: 10.21037/atm-21-4561

View this article at: https://dx.doi.org/10.21037/atm-21-4561 


\section{Introduction}

Metabolic syndrome (MS) refers to the aggregation of risk factors associated with increased risk of cardiovascular disease and diabetes, including abdominal obesity, hypertension, impaired glucose tolerance, insulin resistance, decreased high-density lipoprotein cholesterol (HDL-C), and triglyceride (TG) elevation $(1,2)$. Lu et al. (3) reported that the prevalence of MS was 33.9\% (36.8\% for females and $31 \%$ for males) among 98,658 adults aged $\geq 18$ years in 31 provinces of China, the prevalence of MS in females was higher than that in males. Obesity is one of the core factors of MS and has reached epidemic proportions in many countries (4). Importantly, a previous study found that a large number of substances are secreted in adipose tissue accumulated during obesity. These secretory substances, which include leptin, resistin, inflammatory factors, nonesterified fatty acid (NEFA), and plasminogen activator inhibitor-1 (PAI-1), play an important role in promoting insulin resistance, hypertension, and fatty liver (5). The elevation of TG in the liver results in disturbances of fat metabolism. Dyslipidemia, another distinctive feature of MS, entails increased NEFA content, elevated TG levels, low HDL-C levels, and increased small dense low-density lipoprotein (LDL) and apolipoprotein(apo)B (apoB) levels (6). Research has shown that obesity and insulin resistance are closely related to low inflammatory state in adipose tissue. Thus, resolving the inflammation is one potential strategy to treat metabolic syndromes, and macrophage recruitment and polarization are key to obesity-induced inflammation and insulin resistance (7).

Macrophages can be divided into two distinct phenotypes depending on their mode of activation and function after activation: classically activated M1 macrophages and alternatively activated M2 macrophages. These phenotypes are characterized by the expression of their markers, the production of specific factors, and their biological effects (8). M1-type macrophages have proinflammatory effects (9), while M2-type macrophages have anti-inflammatory effects (10). The M1 phenotype expresses CCL3, CCL5, CD80, and inducible nitric oxide synthase (iNOS) under induction by tumor necrosis factor- $\alpha(\mathrm{TNF}-\alpha)$, interleukin (IL)-1 $\beta$, and IL-23. Meanwhile, the M2 phenotype expresses CD206, CD163, CCL22, and arginase-1 (Arg-1) under stimulation with IL-4, IL-10, TGF- $\beta$, and IL-13 (11). Reddy et al. (12) showed that MS can be characterized by monocytes and tissue macrophages that are activated into the proinflammatory phenotype.
Lupeol is found in vegetables such as white cabbage, cucumber, tomato, in fruits such as olive, fig, mango, strawberry and in medicinal plants such as Shea butter plant, Tamarindus indica, American ginseng and Sebastiania adenophora, see Figure 1 for chemical structure. Various medicinal properties of lupeol have been reported in the literature, including its anti-inflammatory, antioxidant, anti-diabetic, anti-mutagenic, and skin wound repairing effects (13). Lupeol can attenuate the alteration characteristics of allergic airway inflammation (14), and it has also been found to inhibit neuroinflammation in cerebellar cultures and induce neuroprotection related to the modulation of astrocyte response and the expression of neurotrophic and inflammatory factors (15). Preventive administration of diindolylmethane and lupeol acts as a potent Cox-2 inhibitor which activates the tumor suppressor protein phosphotensin (PTEN) to exert antiproliferative and anti-inflammatory effects in experimental bladder carcinogenesis (16). Previous study has demonstrated that lupeol treatment resulted in a switch of macrophages from M1 to M2 phenotype, and that administration of Lupeol to DSS-induced colitis mouse model mitigated intestinal inflammation, leading to increased mouse survival from lethal colitis (17). However, whether lupeol is beneficial for MS has not been investigated thus far. We hypothesize that lupeol may play an anti-inflammatory role through modulating macrophage phenotypes. Therefore, we established the in vivo MS model by feeding rats a high-fat diet (HFD), applied Lupeol to the MS model and evaluated its therapeutic effect, observed the polarization of macrophages, and evaluated the production of cytokines and the expression of cell surface markers. We present the following article in accordance with the ARRIVE reporting checklist (available at https://dx.doi.org/10.21037/atm-21-4561).

\section{Methods}

\section{MS model}

Forty specific-pathogen-free male Sprague Dawley (SD) rats (4 weeks old, 80-100 g) were obtained from the Department of Laboratory Animal Science, Harbin Medical University. The animal experiments began after 1 week of adaptation. The rats were randomly divided into 5 groups: control group, MS model group, lupeol $20 \mathrm{mg} / \mathrm{kg}$ group, lupeol $50 \mathrm{mg} / \mathrm{kg}$ group, and lupeol $100 \mathrm{mg} / \mathrm{kg}$ group. The MS rat model was induced by feeding rats an HFD consisting of $40 \%$ fat $(5.02 \mathrm{kcal} / \mathrm{g})$ for 10 weeks. 


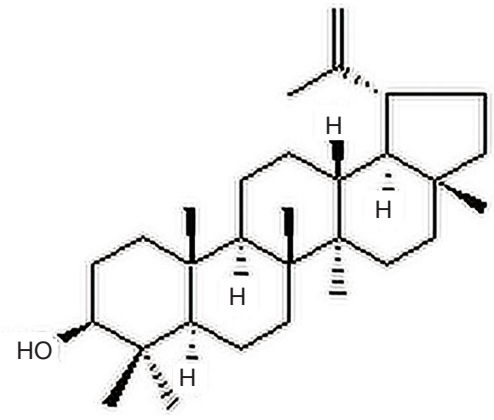

Figure 1 Chemical structure of lupeol.

The rats in the normal control group were fed daily (for growth and maintenance) with a diet containing $3.58 \mathrm{kcal} / \mathrm{g}$. The rats were housed under a 12-hour light/dark cycle at $25 \pm 3{ }^{\circ} \mathrm{C}$ and $60 \%$ humidity, and had free access to water. Compared with the control group, the body weight of rats was significantly increased, FBG was significantly increased, and blood lipid metabolism was abnormal, indicating that the MS model was successfully induced.

Lupeol was purchased from Mansite (Beijing, China). It had purity $>98 \%$ and a molecular weight of $426.72 \mathrm{~g} / \mathrm{mol}$. An aqueous suspension of lupeol was prepared and intraperitoneal injection of $0.1 \mathrm{~mL}$ to rats at 20,50 , or $100 \mathrm{mg} / \mathrm{kg}$ (18) (the lupeol $20 \mathrm{mg} / \mathrm{kg}$, lupeol $50 \mathrm{mg} / \mathrm{kg}$, and lupeol $100 \mathrm{mg} / \mathrm{kg}$ groups respectively). After 28 days of continuous intraperitoneal administration, the regulatory effect of lupeol on MS was observed. Experiments were performed under a project license (No. 2020035) granted by ethics committee of The First Affiliated Hospital of Harbin Medical University, in compliance with Animal Management Rule of the People's Republic of China (Ministry of Health, China, document No. 55,2001). A protocol was prepared before the study without registration.

\section{Obesity index}

A midline incision was made to open the abdomen of each rat. The visceral fat pad was then dissected out and weighed. The amount of visceral fat associated with body weight (\%) was calculated using the following formula (19): obesity index $=$ visceral fat mass $(\mathrm{g}) /$ terminal body mass $(\mathrm{g}) \times 100 \%$.

\section{Blood analysis}

The following serum parameters of the rats were measured: glucose, total cholesterol (TC), TG, LDL, and HDL.
Blood analyses were performed on a Dimension EXL analyzer (Siemens Healthcare Diagnostics s.r.l., Milan, Italy) using commercial reagents. An Advia 2120 blood cell counter (Siemens, Erlangen, Germany) was used to perform routine blood counts on EDTA-treated samples.

\section{Other determinations}

Insulin, IL-6, TNF- $\alpha$, monocyte chemoattractant protein-1 (MCP-1), IL-4, and IL-10 levels were measured using highsensitivity enzyme-linked immunosorbent assay (ELISA) kits. Rat insulin Elisa Kit was purchased from EMD Millipore Corporation (Billerica, MA, USA). The cytokines IL-6, TNF- $\alpha$, MCP-1, IL-4, and IL-10 Elisa Kits were purchased from Nanjing Institute of Bioengineering.

\section{Histology}

The rats were anesthetized and euthanasia by an intraperitoneal injection of sodium pentobarbital (40-60 mg/kg) (20), after which the liver tissue was removed. Rat liver tissue samples were divided into two groups, one group stained with hematoxylin and eosin (HE). The other group samples were stained with Oil Red $\mathrm{O}$ and then re-stained with hematoxylin. Finally, a light microscope was used to observe the histopathological morphology.

\section{Total RNA and quantitative real-time PCR}

Serum from the rats was homogenized in $1 \mathrm{~mL}$ TRIzol (Invitrogen, Beijing, China) and processed for isolation in adherence with the manufacturer's instructions. Samples containing $1 \mu \mathrm{g}$ of total RNA were measured using a Gene Quant ProRNA/DNA Calculator (Amersham Pharmacia Biotec, UK) and then treated with DNaseI in a $10-\mu \mathrm{L}$ reaction system (TakaRa, Dalian, China). The RNA was reverse transcribed into complementary DNA using the Prime Script ${ }^{\mathrm{TM}}$ RT Mix reagent Kit (TakaRa, Dalian, China). $\beta$-actin was amplified as a house keeping gene. Quantitative real-time PCR (qRT-PCR) was performed using SYBR Premix Ex Taq ${ }^{\mathrm{TM}}$ II (TakaRa, Dalian, China) on a Bio-Rad CFX96 system (Bio-Rad, CA, USA). The $2^{-\Delta \Delta \mathrm{Ct}}$ method was employed to calculate the relative mRNA expression levels.

\section{Flow cytometry analysis}

Peripheral blood mononuclear cells (PBMCs) of rats 
in each group were collected from for flow cytometry. PBMCs were added to a tube containing antibody F4/80 and CD206 or F4/80 and iNOS (GeneTex, SC, USA) and dye liquor, and then incubated for 15 minutes in the dark. After the addition of $2 \mathrm{~mL} 1 \times$ lysing solution, the PBMCs were incubated in the dark for a further 30 minutes and then centrifuged at 1,500 rap. Subsequently, the supernatant was removed and $2 \mathrm{~mL}$ phosphate-buffered saline (PBS) was added, after which, centrifugation at 1,500 rap was performed for a second time. Finally, the supernatant was removed and $500 \mu \mathrm{L}$ PBS was added. The samples were analyzed using a FACSAria cell sorter (BD Biosciences). The data were analyzed using FlowJo (Tree Star, Ashland, OR, USA). M1 and M2 macrophages were identified as $\mathrm{F} 4 / 80^{+}$iNOS $^{+}$and $\mathrm{F} 4 / 80^{+} \mathrm{CD} 206^{+}$cells, respectively. Ratio of $\mathrm{F} 4 / 80^{+}{ }^{+} \mathrm{NOS}^{+}$cells or $\mathrm{F} 4 / 80^{+} \mathrm{CD} 206^{+}$cells to the number of negative cells stained with trypan blue to obtain the polarization level of M1 or M2 macrophages (\%).

\section{Western blot}

Total protein was extracted from the heart tissues of the rats. The heart tissues were placed in an EP tube, to which $500 \mu \mathrm{L}$ RIPA lysis buffer containing the protease inhibitor phenylmethylsulfonyl fluoride was added. The heart tissues were pulverized with an ultrasonic disintegrator, then lysed in an ice-water bath for 10-15 seconds, lysed on ice for 45 minutes, and centrifuged at $4{ }^{\circ} \mathrm{C}$ and $12,000 \mathrm{rpm}$ for 15 minutes. The supernatant was aspirated using a clean aspiration method. A bicinchoninic acid (BCA) assay kit (Beyotime Institute of Biotechnology, Shanghai, China) was employed to detect the protein concentration. According to the manufacturer's guidance, the protein with a quality of $20 \mu \mathrm{g} / \mu \mathrm{L}$ was selected for testing. Polyvinylidene difluoride membranes were sealed with $5 \%$ skim milk at $37^{\circ} \mathrm{C}$ for 120 minutes and then incubated with the following primary antibodies at $4{ }^{\circ} \mathrm{C}$ overnight: rabbit anti-actin antibody (1:1,000, ab8227, Abcam), rabbit anti-iNOS (1:250, ab15323, Abcam), and rabbit anti-Arg-1 (1:1,000, ab124917, Abcam). After that, the membranes were incubated with secondary antibodies. The density of the protein bands was determined and analyzed using the Bio-Rad CFX96 system.

\section{Statistical analysis}

All experimental data were expressed as the mean \pm standard deviation (SD). SPSS 22.0 software (SPSS, Chicago, IL, USA) was used to perform all statistical analyses. All data pictures analyses were performed in GraphPad Prism 5.01 (GraphPad Software Inc., La Jolla, CA, USA). Differences with $\mathrm{P}<0.05$ were considered significant.

\section{Results}

\section{The effects of lupeol on physiological and biochemical indexes in the MS model}

As shown in Figure 2, the fasting blood glucose levels, body weight, obesity index, and fasting serum insulin levels of the MS model rats were significantly increased compared to those of the control group $(\mathrm{P}<0.05)$, indicating that the MS model was successfully induced. However, after treatment with lupeol (at 20,50, or $100 \mathrm{mg} / \mathrm{kg}$ ), the fasting blood glucose levels, body weight, obesity index, and fasting serum insulin levels were significantly decreased in a dosedependent manner compared to those observed in the MS model group $(\mathrm{P}<0.05)$.

\section{The effects of lupeol on pathological liver injury and the expression of inflammatory factors in the MS model}

Research has shown that the novel paradigm of nonalcoholic fatty liver disease is a strong determinant for the development of MS (21). As shown in Figure 3, HE staining revealed an increased number of fat droplets, a large lesion range, concentrated nuclei, and more necrotic cells in the livers of the MS model rats compared with the control group. Observation of the pathological sections showed that the number of lipid droplet cells in the livers of the MS model rats decreased as the lupeol dose increased. Furthermore, in the MS model rats, the protein expression levels of TNF- $\alpha$, IL-6, MCP-1, IL-4, and IL-10 in the liver were markedly upregulated relative to those in the control group $(\mathrm{P}<0.05)$. However, after treatment with medium and high doses of lupeol (50 and $100 \mathrm{mg} / \mathrm{kg}$, respectively), there were marked declines in the levels of the proinflammatory factors TNF- $\alpha$, IL-6, and MCP-1 in comparison with the MS model group $(\mathrm{P}<0.05)$; in contrast, the levels of the anti-inflammatory factors IL-4 and IL-10 were markedly enhanced $(\mathrm{P}<0.05)$.

\section{The effects of lupeol on lipid metabolism indicators in the MS model}

As shown Figure 4, following Oil Red O staining, only lightblue nuclei were seen in the liver tissue sections of normal 

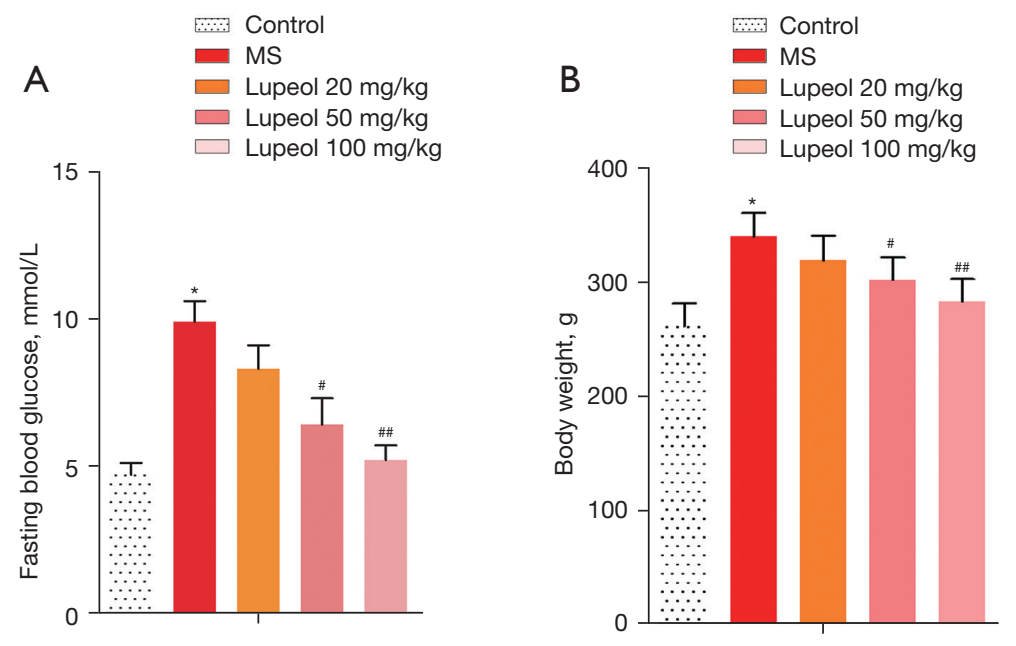

C

$\mathrm{D}$
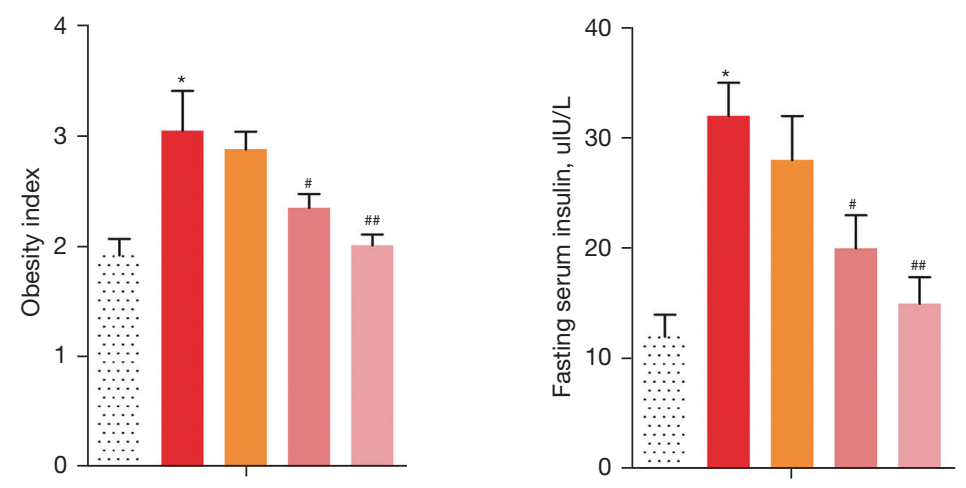

Figure 2 The effects of lupeol on physiological and biochemical indexes in the MS model. Forty Sprague Dawley rats were randomly divided into 5 groups: control group, MS model group, lupeol $20 \mathrm{mg} / \mathrm{kg}$ group, lupeol $50 \mathrm{mg} / \mathrm{kg}$ group, and lupeol $100 \mathrm{mg} / \mathrm{kg}$ group. (A) Fasting blood glucose (mmol/L), detected using the Dimension EXL analyzer; (B) body weight (g); (C) obesity index [obesity index = visceral fat mass $(\mathrm{g}) /$ terminal body mass $(\mathrm{g}) \times 100 \%$; and $(\mathrm{D})$ fasting serum insulin (uIU/L), detected by ELISA. $\left(^{*}, \mathrm{P}<0.05\right.$ versus control group; ", $\mathrm{P}<0.05$, \#\#, $\mathrm{P}<0.01$ versus MS group). MS, metabolic syndrome; ELISA, enzyme-linked immunosorbent assay.

rats, and no red-stained lipid droplets were observed. The MS model group exhibited a large number of irregular red lipid droplets; however, in the lupeol $100 \mathrm{mg} / \mathrm{kg}$ group, there were no red-stained lipid droplets, and light-blue nuclei were observed.

Aside from obesity and insulin resistance, dyslipidemia is another distinctive feature of MS. The main indicators of lipid metabolism include TC, TG, LDL, and HDL in the serum. In the MS model rats, the levels of TC, TG, and LDL were obviously elevated compared with those in the control group $(\mathrm{P}<0.05)$, while the level of HDL was obviously downregulated $(\mathrm{P}<0.05)$. After treatment with medium and high doses of lupeol $(50 \mathrm{mg} / \mathrm{kg}$ and $100 \mathrm{mg} / \mathrm{kg}$, respectively), the levels of TC, TG, and LDL displayed marked declines relative to those in the MS model group $(\mathrm{P}<0.05)$, whereas the level of HDL was obviously upregulated $(\mathrm{P}<0.05)$.

\section{Lupeol treatment inbibits M1 polarization but promotes M2 polarization of macrophages}

Macrophages are well known to have the ability to switch between the M1 and M2 types in the event of injury. Polarization of adipose tissue macrophages plays a critical role in the pathological progression of obesity (22). As shown in Figure 5, M1 macrophages $\left(\mathrm{F} 4 / 80^{+} \mathrm{iNOS}^{+}\right)$and $\mathrm{M} 2$ macrophages $\left(\mathrm{F} 4 / 80^{+} \mathrm{CD} 206^{+}\right.$) were remarkably enhanced in MS model rats compared to the control group $(\mathrm{P}<0.05)$. 


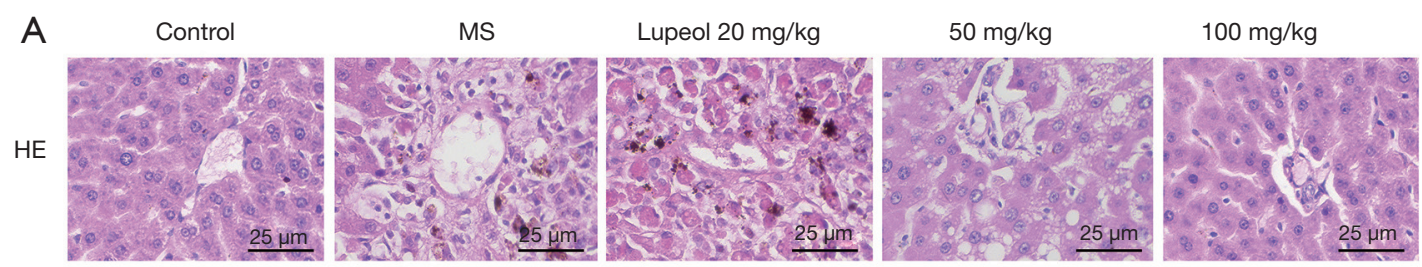

B
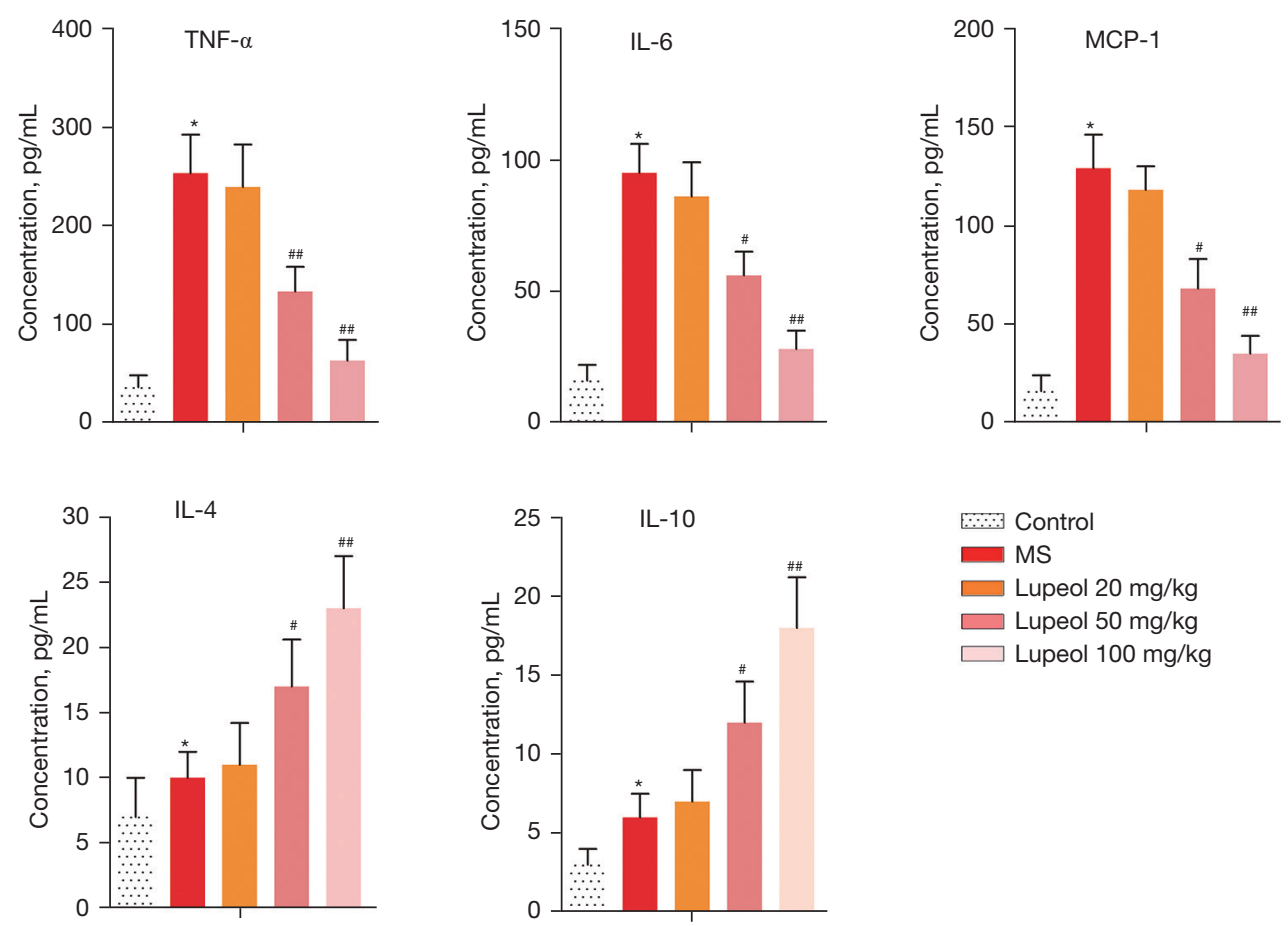

$$
\begin{aligned}
& \square \text { Control } \\
& \text { MS } \\
& \text { Lupeol } 20 \mathrm{mg} / \mathrm{kg} \\
& \square \text { Lupeol } 50 \mathrm{mg} / \mathrm{kg} \\
& \square \text { Lupeol } 100 \mathrm{mg} / \mathrm{kg}
\end{aligned}
$$

Figure 3 The effects of lupeol on pathological liver injury and the expression of inflammatory factors in the MS model. (A) HE staining results show an increased number of fat droplets, a large lesion range, concentrated nuclei, and necrotic cells in the livers of the MS model rats. Magnification 400×. (B) The protein expression levels of inflammatory factors (TNF- $\alpha$, IL-6, MCP-1, IL-4, and IL-10) in the liver were detected using enzyme-linked immunosorbent assays. ( ${ }^{*}, \mathrm{P}<0.05$ versus control group; ${ }^{\#}, \mathrm{P}<0.05,{ }^{\#}, \mathrm{P}<0.01$ versus $\mathrm{MS}$ group). MS, metabolic syndrome; HE, hematoxylin and eosin; TNF, tumor necrosis factor; IL, interleukin; MCP-1, monocyte chemoattractant protein-1.

Interestingly, M1 macrophages $\left(\mathrm{F} 4 / 80^{+}{ }^{i N O S}{ }^{+}\right)$were remarkably inhibited in lupeol-treated rats compared to the MS model group $(\mathrm{P}<0.05)$. Meanwhile, M2 macrophages $\left(\mathrm{F} 4 / 80^{+} \mathrm{CD} 206^{+}\right)$were remarkably elevated following lupeol treatment $(\mathrm{P}<0.05)$. These results demonstrate that lupeol treatment promotes $\mathrm{M} 2$ polarization while inhibiting M1 polarization.

To further assess whether lupeol can regulate macrophages polarization, M1 markers and M2 markers were detected in peripheral blood by qRT-PCR. As shown in Figure 6, the levels of M1 markers (iNOS, IL-1 1 , IL-6 and TNF- $\alpha$ ) were significantly elevated in the MS model group compared with the control group $(\mathrm{P}<0.05)$, whereas the levels of M2 markers (Arg-1, IL-10, CD206 and TGF- $\beta$ ) were suppressed $(\mathrm{P}<0.05)$. After treatment with lupeol, the levels of M1 markers (iNOS, IL-1 $\beta$, IL-6 and TNF- $\alpha$ ) were markedly inhibited compared with the MS model group $(\mathrm{P}<0.05)$, while those of $\mathrm{M} 2$ markers (Arg1, IL-10, CD206 and TGF- $\beta$ ) were markedly enhanced $(\mathrm{P}<0.05)$. For iNOS and Arg-1, the results of western blot analysis were consistent with those of the qRT-PCR analysis. Taken together, these data demonstrate that lupeol polarized macrophages away from the M1 phenotype and toward an M2-like state in MS. 
A

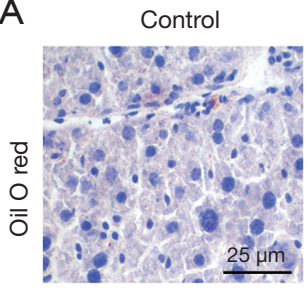

MS

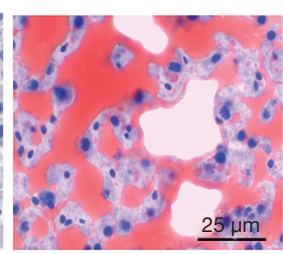

Lupeol 20 mg/kg

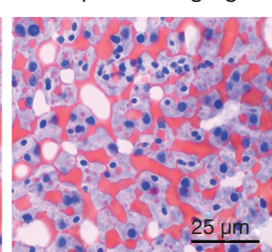

$50 \mathrm{mg} / \mathrm{kg}$

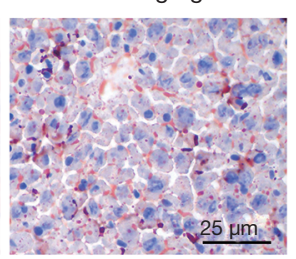

$100 \mathrm{mg} / \mathrm{kg}$

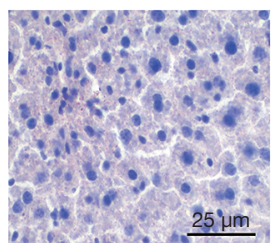

B

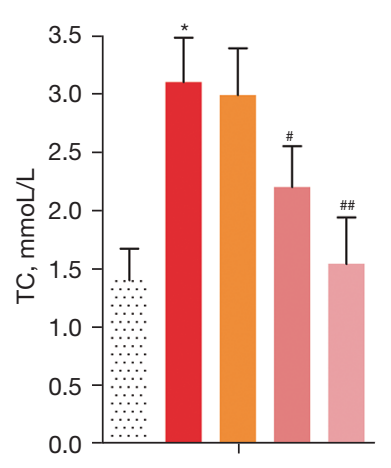

D

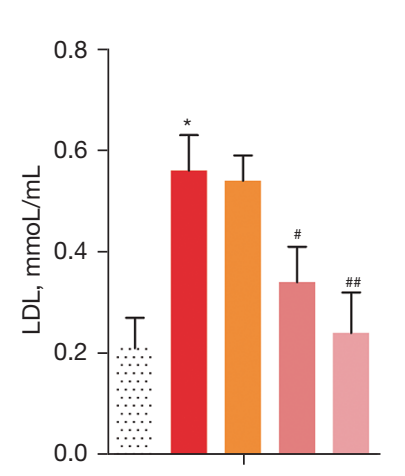

C

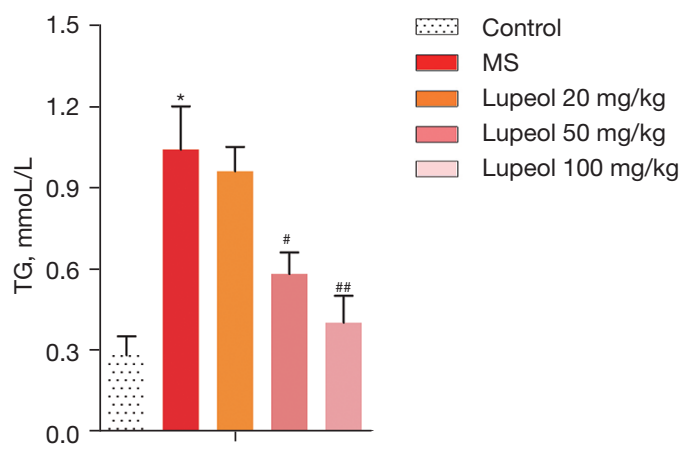

E

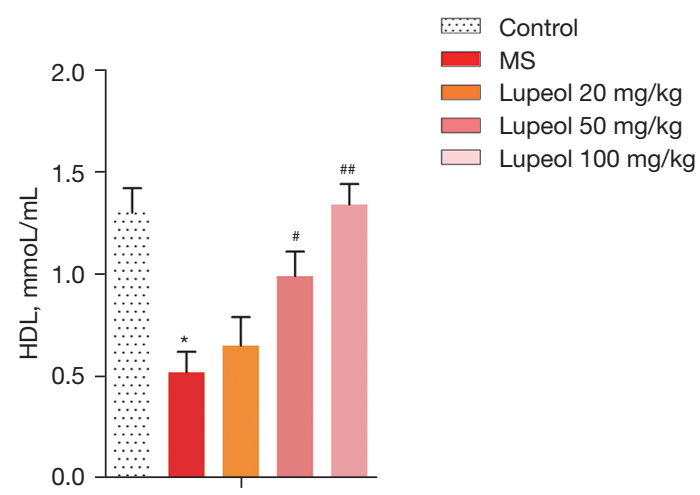

Figure 4 The effects of lupeol on lipid metabolism indicators in the MS model (A) Oil Red O staining results show only light-blue nuclei in the liver tissue sections of normal rats, with no red-stained lipid droplets observed, and a large number of irregular red lipid droplets in the MS model group. Magnification 400x. (B) TC (mmol/L), (C) TG (mmol/L), (D) LDL (mmol/mL), and (E) HDL (mmol/L). Indicators (TC,

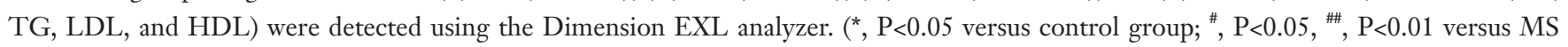
group). MS, metabolic syndrome; TC, total cholesterol; TG, triglyceride; LDL, low-density lipoprotein; HDL, high-density lipoprotein.

\section{Discussion}

MS is a group of cardiometabolic risk factors and comorbidities that carry high risks of cardiovascular disease and type 2 diabetes. MS causes morbidity and mortality in many countries, and entails huge socio-economic costs (23). The objective of the present study was to determine whether lupeol could inhibit the inflammatory mediators associated with the regulation of macrophage phenotypes and functions in rats with MS. The results showed that body weight, obesity index, fasting blood glucose, and serum were increased significantly in rats fed an HFD. It has been increasingly recognized that an HFD not only induces obesity, diabetes, and dyslipidemia in mice, but also causes pathological changes in the liver $(24,25)$. The results of HE staining showed that the number of fat droplets was increased, the lesion range was large, the nuclei were concentrated, and more cells were necrotic in the livers of the MS model group compared with the control group. At the same time, Oil Red O staining revealed that the MS model group exhibited a large number of irregular red lipid droplets. These results further indicate that metabolic 

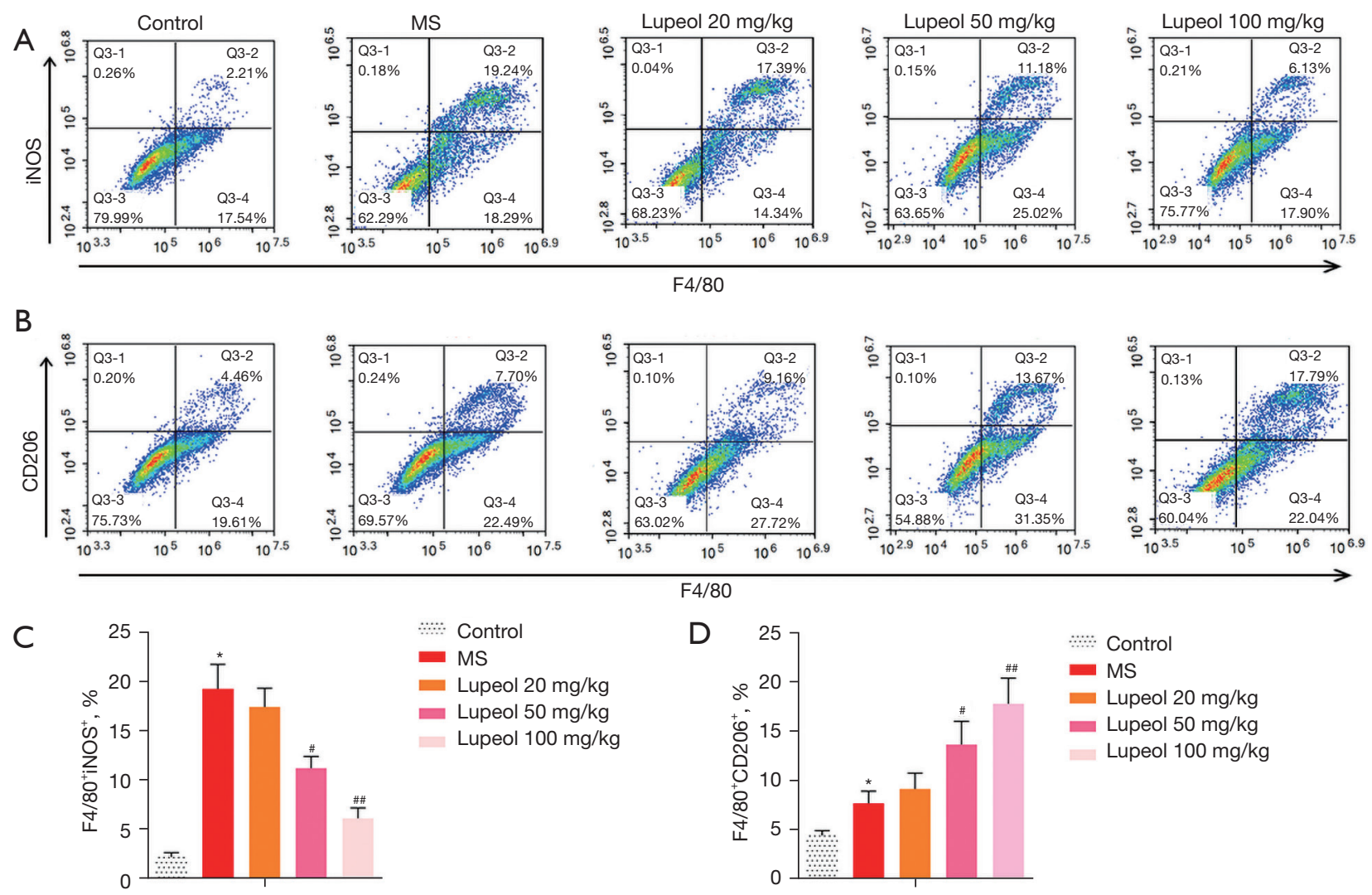

Figure 5 Lupeol treatment inhibits M1 polarization but promotes M2 polarization of macrophages (A) Representative flow cytometry

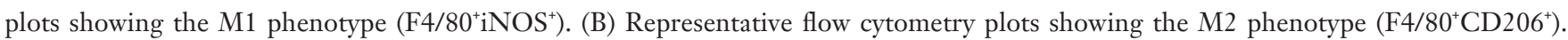
(C) Quantification of results from A. (D) Quantification of results from B. ( ${ }^{*}, \mathrm{P}<0.05$ versus control group; ${ }^{\#}, \mathrm{P}<0.05$, ,, $\mathrm{P}<0.01$ versus MS group). MS, metabolic syndrome; iNOS, inducible nitric oxide synthase.

synthesis aggravates hepatic steatosis and obesity.

Inflammation is one of the underlying causes of MS (2). In this study, the expression levels of TNF- $\alpha$, IL-6, MCP-1, IL-4, and IL-10 protein in the livers of MS mice were markedly upregulated compared to those in the control group. Adipose tissue responds to overnutrition by mounting an immune response and can release large amounts of cytokines such as TNF- $\alpha$ and IL-6 (26). After treatment with lupeol, there were marked declines in the levels of proinflammatory factors (TNF- $\alpha$, IL-6, and MCP-1) compared with the MS model group. Previous studies have shown that lupeol induces the downregulation of the mRNA expression of the proinflammatory markers TNF, iNOS, and NLRP3 (15), and inhibits the elevation of digestive enzymes and cytokines, such as TNF- $\alpha$, IL-1, and IL-6 (27). This suggests that lupeol can enhance the anti-inflammatory response and reduce the excessive inflammatory injury to the body caused by MS.
Dyslipidemia is a distinctive feature of MS and includes increased NEFA content, elevated TG levels, low HDL-C levels, and increased small dense (LDL) and apoB levels (6). In our study, the levels of TC, TG, and LDL were markedly higher in MS model group than those in the control group, while the level of HDL was significantly down-regulated. However, after lupeol treatment, the levels of TC, TG, and LDL were obviously decreased compared with those in the MS model group, while the level of HDL was obviously up-regulated. Semenkovich reported that one of the main characteristics associated with lipid metabolism is the increase of NEFA with the context of insulin resistance (28), and when there is too much NEFA in the circulation, NEFA flows into the liver. If the NEFA flowing into the liver increased, the production of TG and glucose in the liver increased, and excessive TG was secreted in the form of LDL (29). Together, these observations suggest that lupeol could weaken injury caused by dyslipidemia in the 
$\mathrm{A}$
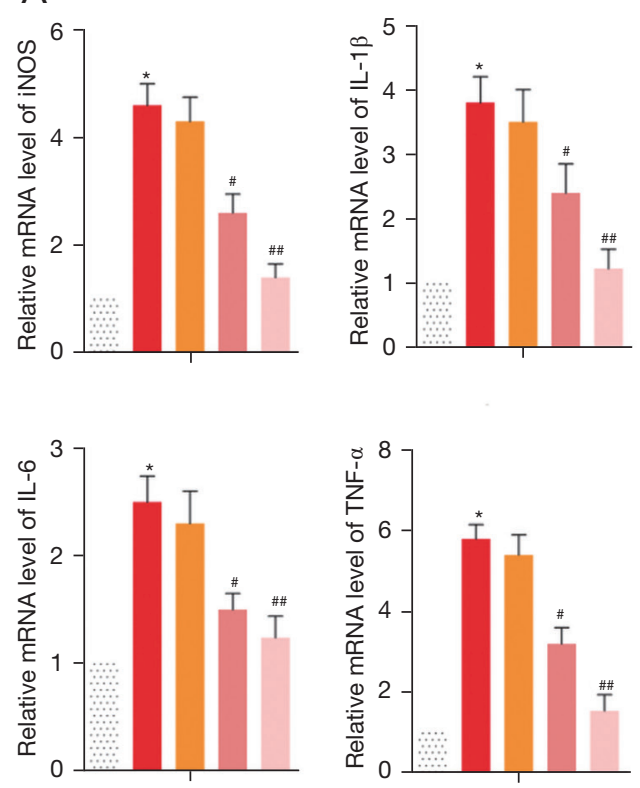

B
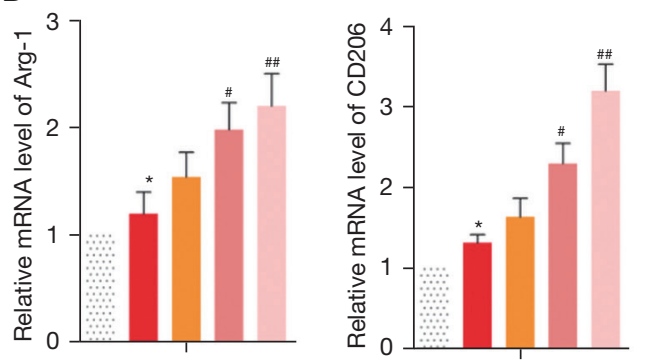

…... Control

- MS

— Lupeol 20 mg/kg

Lupeol 50 mg/kg

$\square$ Lupeol $100 \mathrm{mg} / \mathrm{kg}$
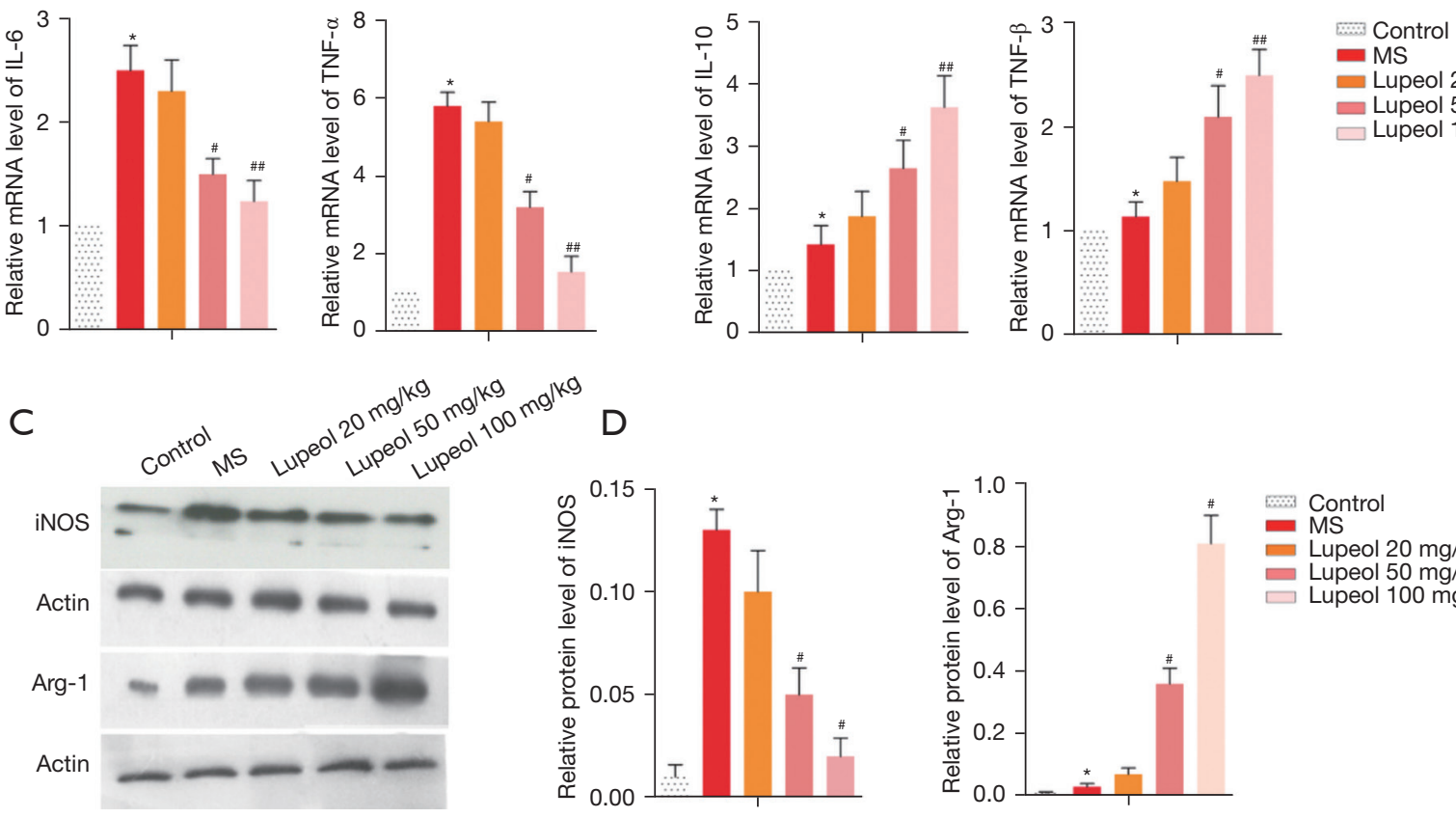

- Lupeol 20 mg/kg - Lupeol 50 mg/kg $\square$ Lupeol 100 mg/kg

\section{.}




\section{Page 10 of 11}

polarized macrophages away from the M1 phenotype and toward an M2-like state in rats with MS.

\section{Conclusions}

In summary, this study has shown that lupeol treatment can markedly reduce fasting blood glucose levels, body weight, obesity index, and fasting serum insulin levels, while repairing liver injury, restoring the blood lipid balance, and significantly increasing the production of anti-inflammatory factors in rats with MS. Furthermore, it promotes macrophage switching from the M1 to the M2 phenotype. Taken together, our findings suggest a potential role of lupeol as a therapeutic agent for MS.

\section{Acknowledgments}

Funding: None.

\section{Footnote}

Reporting Checklist: The authors have completed the ARRIVE reporting checklist. Available at https://dx.doi. org/10.21037/atm-21-4561

Data Sharing Statement: Available at https://dx.doi. org/10.21037/atm-21-4561

Conflicts of Interest: All authors have completed the ICMJE uniform disclosure form (available at https://dx.doi. org/10.21037/atm-21-4561). The authors have no conflicts of interest to declare.

Ethical Statement: The authors are accountable for all aspects of the work in ensuring that questions related to the accuracy or integrity of any part of the work are appropriately investigated and resolved. Experiments were performed under a project license (No. 2020035) granted by ethics committee of The First Affiliated Hospital of Harbin Medical University, in compliance with Animal Management Rule of the People's Republic of China (Ministry of Health, China, document No. 55,2001).

Open Access Statement: This is an Open Access article distributed in accordance with the Creative Commons Attribution-NonCommercial-NoDerivs 4.0 International License (CC BY-NC-ND 4.0), which permits the noncommercial replication and distribution of the article with the strict proviso that no changes or edits are made and the original work is properly cited (including links to both the formal publication through the relevant DOI and the license). See: https://creativecommons.org/licenses/by-nc-nd/4.0/.

\section{References}

1. Fiszman M, Rosemblat $G$, Ahlers CB, et al. Identifying risk factors for metabolic syndrome in biomedical text. AMIA Annu Symp Proc 2007;2007:249-53.

2. O'Neill S, O'Driscoll L. Metabolic syndrome: a closer look at the growing epidemic and its associated pathologies. Obes Rev 2015;16:1-12.

3. Lu J, Wang L, Li M, et al. Metabolic Syndrome Among Adults in China: The 2010 China Noncommunicable Disease Surveillance. J Clin Endocrinol Metab 2017;102:507-15.

4. Jastreboff AM, Kotz CM, Kahan S, et al. Obesity as a disease: The Obesity Society 2018 Position Statement. Obesity (Silver Spring) 2019;27:7-9.

5. Haluzík M, Trachta P, Haluzíková D. Adipose tissue hormones. Vnitr Lek 2010;56:1028-34.

6. Ginsberg HN, Huang LS. The insulin resistance syndrome: impact on lipoprotein metabolism and atherothrombosis. J Cardiovasc Risk 2000;7:325-31.

7. Yang J, Ran Y, Yang Y, et al. 4-Hydroxyisoleucine alleviates macrophage-related chronic inflammation and metabolic syndrome in mice fed a high-fat diet. Front Pharmacol 2021;11:606514.

8. Shapouri-Moghaddam A, Mohammadian S, Vazini H, et al. Macrophage plasticity, polarization, and function in health and disease. J Cell Physiol 2018;233:6425-40.

9. Gordon S, Taylor PR. Monocyte and macrophage heterogeneity. Nat Rev Immunol 2005;5:953-64.

10. Gordon S. Alternative activation of macrophages. Nat Rev Immunol 2003;3:23-35.

11. Gao S, Li C, Zhu Y, et al. PEDF mediates pathological neovascularization by regulating macrophage recruitment and polarization in the mouse model of oxygen-induced retinopathy. Sci Rep 2017;7:42846.

12. Reddy P, Lent-Schochet D, Ramakrishnan N, et al. Metabolic syndrome is an inflammatory disorder: A conspiracy between adipose tissue and phagocytes. Clin Chim Acta 2019;496:35-44.

13. Pereira Beserra F, Xue M, Maia GLA, et al. Lupeol, a Pentacyclic Triterpene, Promotes Migration, Wound Closure, and Contractile Effect In Vitro: Possible Involvement of PI3K/Akt and p38/ERK/MAPK Pathways. 
Molecules 2018;23:2819.

14. Vasconcelos JF, Teixeira MM, Barbosa-Filho JM, et al. The triterpenoid lupeol attenuates allergic airway inflammation in a murine model. Int Immunopharmacol 2008;8:1216-21.

15. Oliveira-Junior MS, Pereira EP, de Amorim VCM, et al. Lupeol inhibits LPS-induced neuroinflammation in cerebellar cultures and induces neuroprotection associated to the modulation of astrocyte response and expression of neurotrophic and inflammatory factors. Int Immunopharmacol 2019;70:302-12.

16. Prabhu B, Balakrishnan D, Sundaresan S. Antiproliferative and anti-inflammatory properties of diindolylmethane and lupeol against N-butyl-N-(4-hydroxybutyl) nitrosamine induced bladder carcinogenesis in experimental rats. Hum Exp Toxicol 2016;35:685-92.

17. Zhu Y, Li X, Chen J, et al. The pentacyclic triterpene Lupeol switches M1 macrophages to M2 and ameliorates experimental inflammatory bowel disease. Int Immunopharmacol 2016;30:74-84.

18. de Lima FO, Alves V, Barbosa Filho JM, et al. Antinociceptive effect of lupeol: evidence for a role of cytokines inhibition. Phytother Res 2013;27:1557-63.

19. Gumede NM, Lembede BW, Brooksbank RL, et al. $\beta$-Sitosterol Shows Potential to Protect Against the Development of High-Fructose Diet-Induced Metabolic Dysfunction in Female Rats. J Med Food 2020;23:367-74.

20. Fan S, Sun JB, Li R, et al. Lycopene protects myocardial ischemia injury through anti-apoptosis and anti-oxidative stress. Eur Rev Med Pharmacol Sci 2019;23:3096-104.

21. Lonardo A, Ballestri S, Marchesini G, et al. Nonalcoholic fatty liver disease: a precursor of the metabolic syndrome. Dig Liver Dis 2015;47:181-90.

22. Lumeng CN, Bodzin JL, Saltiel AR. Obesity induces a phenotypic switch in adipose tissue macrophage polarization. J Clin Invest 2007;117:175-84.

23. Zimmet P, Alberti KGMM, Stern N, et al. The Circadian Syndrome: is the Metabolic Syndrome and much more! J Intern Med 2019;286:181-91.

24. Dorn C, Engelmann JC, Saugspier M, et al. Increased expression of c-Jun in nonalcoholic fatty liver disease. Lab Invest 2014;94:394-408.

25. Dorn C, Riener MO, Kirovski G, et al. Expression of fatty acid synthase in nonalcoholic fatty liver disease. Int J Clin Exp Pathol 2010;3:505-14.

26. Reilly SM, Saltiel AR. Adapting to obesity with adipose tissue inflammation. Nat Rev Endocrinol 2017;13:633-43.

27. Kim MJ, Bae GS, Choi SB, et al. Lupeol Protects Against Cerulein-Induced Acute Pancreatitis in Mice. Phytother Res 2015;29:1634-9.

28. Semenkovich CF. Fatty acid metabolism and vascular disease. Trends Cardiovasc Med 2004;14:72-6.

29. $\mathrm{Na} \mathrm{HY}$, Lee BC. Scutellaria baicalensis alleviates insulin resistance in diet-induced obese mice by modulating inflammation. Int J Mol Sci 2019;20:727.

30. Stout RD, Jiang C, Matta B, et al. Macrophages sequentially change their functional phenotype in response to changes in microenvironmental influences. J Immunol 2005;175:342-9.

(English Language Editor: J. Reynolds)
Cite this article as: Li J, Huang Y, Han Y, Wang J, Zhang C, Jiang J. Lupeol reduces M1 macrophage polarization to attenuate immunologic dissonance and fatty acid deposition in rats with diet-induced metabolic syndrome. Ann Transl Med 2021;9(20):1534. doi: 10.21037/atm-21-4561 